\title{
Trivium
}

Revue franco-allemande de sciences humaines et sociales - Deutsch-französische Zeitschrift für Geistesund Sozialwissenschaften

$10 \mid 2012$

Lisibilité

\section{Die absolute Lektüre: Theorie und Praxis der christlichen Mystiker im 16. und 17. Jahrhundert}

\section{Michel de Certeau}

Traducteur : Andreas Pfeuffer

\section{(2) OpenEdition}

\section{Journals}

Édition électronique

URL : http://journals.openedition.org/trivium/4147

ISSN : 1963-1820

\section{Éditeur}

Les éditions de la Maison des sciences de l'Homme

\section{Référence électronique}

Michel de Certeau, «Die absolute Lektüre: Theorie und Praxis der christlichen Mystiker im 16. und 17. Jahrhundert », Trivium [Online], 10 | 2012, online erschienen am 30 März 2012, abgerufen am 07 September 2020. URL : http://journals.openedition.org/trivium/4147

Ce document a été généré automatiquement le 7 septembre 2020.

\section{(c) (i) $\odot$}

Les contenus des la revue Trivium sont mis à disposition selon les termes de la Licence Creative Commons Attribution - Pas d'Utilisation Commerciale - Pas de Modification 4.0 International. 


\title{
Die absolute Lektüre: Theorie und Praxis der christlichen Mystiker im 16. und 17. Jahrhundert
}

\author{
Michel de Certeau
}

Traduction : Andreas Pfeuffer

\section{NOTE DE L'ÉDITEUR}

Wir danken Luce Giard für die freundliche Genehmigung, diesen Artikel zu übersetzen.

1 Mit der Lektüre, die ich als "absolut «, im Sinne von abwesend oder losgelöst, bestimme, soll nicht jegliche Art von Lektüre bezeichnet sein. Diese »Losgelöstheit« bezieht sich nur auf eine unter vielen Varianten des Lesens, und sie erlaubt mir, auf einen Aspekt der Beziehung, die wir zum Buch, diesem Garten geordneter Zeichen, unterhalten, näher einzugehen: Was bewerkstelligt der Leser mit diesem mit Schriftzeichen tätowierten Ding?

\section{Die Loslösung des Lesers}

2 Wenn ich mich hier mit der Tätigkeit des Lesens befasse, siedle ich mich im Umfeld zahlreicher Forschungen an. ${ }^{1}$ Es mag genügen, kurz die Ergebnisse der historischen Leseforschung sowie der aus der pädagogischen Psychologie stammenden Untersuchungen des Leseakts in Erinnerung zu rufen.

Zum einen haben die von François Furet und Jacques Ozouf geleiteten Untersuchungen ${ }^{2}$ gezeigt, dass der Erwerb des Lesens lange Zeit über relativ autonom geblieben ist. Bis weit ins 19. Jahrhundert hinein (bis 1868, dem Jahr des Plan d'Étude von Octave Gréard) hat das "Nur-Lesen" eine eigene, von der des Schreibens zu unterscheidende Geschichte. Diese Tradition scheint erst durch die Schule mit dem Erlernen des Schreibens systematisch zusammengeführt worden zu sein. Und auch nachdem die 
Verbindung von Lesen und Schreiben hergestellt ist, bleibt sie zunächst noch wenig gefestigt. Das gilt sowohl für die Vergangenheit als auch für die Gegenwart. Sie bricht etwa beim Abgang von der Schule wieder ab, und viele, die eine Schulbildung genossen haben, bleiben zwar weiterhin Leser, stellen aber das Schreiben ein. Die Geschichte dieser schwierigen und brüchigen Allianz zeigt zumindest das Jahrhunderte lange Fortbestehen einer Lesepraxis, die ohne das Schreiben auskommt und für die dieses auch nicht die Norm darstellt. Sie gibt zudem Anlass, auch und gerade dort über die eigenständigen Entwicklungen des Lesens nachzudenken, wo es eng an das Schreiben gebunden ist.

Die psycholinguistischen Forschungen zum Verstehen ${ }^{3}$ ihrerseits unterscheiden beim Lesen zwischen dem »Leseakt« und dem »Schreibakt«: Das Schulkind lernt gleichzeitig $\mathrm{zu}$ lesen und zu entziffern, und doch leitet sich Ersteres keineswegs aus Letzterem ab. Denn tatsächlich hat das Erkennen oder Buchstabieren von Zeichen nichts mit Lesen zu tun. Es ist vielmehr ein aus dem mündlichen Austausch sich ergebendes und mit dem Hören zusammenhängendes kulturelles Gedächtnis, mit dem der Leser den Text unter Einsatz von Strategien semantischen Abfragens angehen kann. Das entzifferte Schriftstück antwortet nur auf diese Sinnerwartungen; es korrigiert und verfeinert sie. Die Konstruktion hypothetischer Bedeutungen scheint so etwas wie ein Marmorblock zu sein, der im Zuge des Entschlüsselns von graphischem Material nach und nach ausgemeißelt, verschoben, präzisiert oder verifiziert wird, wobei Formen der Lektüre zutage treten, so als ob das Schriftzeichen in eine Antizipation von Sinn hineinmeißeln würde. Die Lektüre folgt demselben Gesetz wie das wissenschaftliche Experiment: Sie ist umso ergiebiger, je zahlreicher die Erwartungen und a priori aufgestellten Hypothesen sind.

5 Von diesen Untersuchungen möchte ich für das, was ich sagen will, Folgendes festhalten: Das Lesen tritt aus der Geschichte des Buches heraus, in der es lange Zeit unterschiedslos aufging. Es war der Leitgedanke der Aufklärung, dass vorrangig das Buch der Erzieher des Volkes ist. Der Leser galt nur als eine Wirkung des Buches. Heute löst er sich von diesen Büchern, auf deren bloßen Schatten man ihn reduzierte. Und nun kappt dieser Schatten just die Verbindung, bekommt ein Profil für sich, gewinnt an Eigenständigkeit.

6 Diese »Loslösung « des Lesers wird von der mystischen Literatur in der Phase gepriesen, in der die mit einem riesigen, von Gott geschriebenen und zum Sprechen gebrachten Buch identifizierte mittelalterliche kosmische Ordnung der Moderne das Feld überlässt, das heißt einer schriftbasierten, Wissen und Geschichte neu organisierenden Ökonomie. Diese Übergangszeit zeichnet sich auch durch eine Relativierung, ja eine Krise der durch das Buch gebildeten Rahmensetzungen aus - der Kosmos wird nicht mehr dem BUCH gleichgesetzt, und der Mythos der buchgestützten Bildung hat seine dominante Position noch nicht erlangt. Sie lässt Raum für die Emanzipation des Lesers als Subjekt. So tritt uns die historische Figur einer Lektüre entgegen, die in ihrer mystischen Form als »absolut« bezeichnet werden kann.

\section{Das Buch - Ort für ein Sagen}

7 Unter »mystisches Lesen« verstehe ich all die Leseverfahren, die während des 16. und 17. Jahrhunderts im Erfahrungsfeld der als "Erleuchtete«, »Mystiker« oder "Spirituelle« bezeichneten Einzelpersonen oder Kollektive empfohlen oder praktiziert 
wurden. Diese Praxis ist sicherlich auf marginale Gruppen beschränkt, jedoch haben diese ein sehr weit verbreitetes praxisorientiertes wie theoretisches Schrifttum hervorgebracht. Die in diesen Texten wiedergegebene Erfahrung vereinigt verschiedene Einzelmerkmale, die hier nur kurz aufgezählt werden sollen: die Opposition gegenüber einem professionalisierten Wissen, das meistens als theologisches Wissen auftritt; eine Infragestellung der für "verderbt « und reformbedürftig gehaltenen Institutionen der Sinngebung; der Wille zum Exil, der sich als Pioniertum in die Regionen des Symbolischen und/oder Imaginären darstellt; ein rastloses Verlangen, gesteigert noch durch die Suche nach dem Anderen oder nach etwas anderem, einem fehlenden Empfänger, dem unbekannten Objekt der Liebe; der Rückgriff auf sämtliche Ausdruckweisen eines erotisierten und theatralisierten Körpers, der von allem, was sich einer Benennung oder sprachlichen Definition entzieht, getroffen, verwundet und entstellt wird. Von dieser "mystischen" Konstellation ist uns eine eigenständige Literatur überliefert. Sie enthält eine Menge von Informationen hinsichtlich der Leseweisen; damit stellt sie zugleich auch Regeln auf, wie sie selbst gelesen werden will.

8 Manche der historischen Postulate dieser Lektüre geben schon Hinweise auf ihren Status und ihre Funktionsweise.

1) Das Buch tritt an die Stelle der als dekadent empfundenen Institutionen oder ihrer Vertreter, die gerade ihre Wissenschaft unempfänglich macht für die Bedürfnisse der "Spirituellen«. Johannes vom Kreuz erklärt, dass er schreibt, da die Seelenführer die Sprache des Verlangens nicht mehr »begreifen« und »verstehen «. ${ }^{4}$ Ebenso Teresa von Ávila, die in den spirituellen Werken das sucht, was ihr die Theologen über die Liebe nicht zu sagen vermögen. ${ }^{5}$ Das Buch tritt an die Stelle der Institution. Es nimmt deren Platz ein. In vielen Fällen ist es überhaupt die Institution.

9 2) Zugleich ändert es seine Beschaffenheit. Während es im Mittelalter in Gestalt der Bibel oder der Ordensregel wesentlicher Bestandteil der Institution selbst war, die für seine Auslegung zuständig war und für die es zugleich als organischer Bestandteil wie als Repräsentation fungierte, erfährt es im 16. Jahrhundert in dreierlei Hinsicht einen Autonomisierungsschub: Seine Verbreitung erfolgt nun in gedruckter Form, die nicht mehr allein von den »Meistern« abhängig ist, der Verfälschung der Texte ein Ende macht und es ermöglicht, sich die in Umlauf gebrachten "Autoren« persönlich anzueignen ${ }^{6}$; die Lektüre wird zu etwas Individuellem und Privatem; schließlich kommt es zu einer inhaltlichen Spezialisierung, aus der eine eigene Gattung, das »spirituelle Buch", hervorgeht, das sich zunehmend von der institutionalisierten theologischen Produktion abnabelt, so wie sich der Roman später von den wissenschaftlichen Werken löst. Dieses neue Buch unterhält nun keine Verbindung mehr mit dem Kosmos, die in Gestalt eines Spiels von Ähnlichkeiten und Durchlässigkeiten mit dem Universum und den gesellschaftlichen Instanzen bestand. Es wird $\mathrm{zu}$ etwas Insulärem, zu einem textuellen Isolat gleichsam, von dem aus sich eigene Wege verfolgen, unbenannte Wünsche verknüpfen, kollektiver Austausch und Übereinkünfte bewerkstelligen lassen. Es wird zu einer pädagogischen Waffe für die Institutionen der Sinnstiftung und vermittlung, steht jedoch weiterhin in Wettbewerb mit der Macht der Mündlichkeit und der Autorität. Ihnen wird das öffentlich zugängliche Schriftstück zu etwas Suspektem und Gefährlichen.

10 3) Der durch das Buch gebildete Ort lässt sich mit dem vergleichen, den »die Gedächtniskunst« seit der Antike in Gestalt eines mentalen Feldes organisierte, in dem 
die Wissensbestandteile derart klassifiziert und angeordnet waren, dass sie sich intellektuell und diskursiv handhaben ließen. ${ }^{7}$ Von diesen Techniken, die aus dem Gedächtnis ein "Theater geistiger Operationen « machten, unterscheidet sich das Buch in mindestens zweierlei Hinsicht: Zum einen verwandelt sich der innere, mentale Ort zu einem dem Subjekt äußerlichen Gegenstand, das, indem es zu seinem Wissen auf Distanz geht, besser imstande ist, sich davon zu unterscheiden und es zu beherrschen, damit zu hantieren oder Kombinationen vorzunehmen. Zum anderen verknüpfte die Gedächtniskunst jeden Figuren- (oder Ideen-)Typus mit einem bestimmten Sitz, wo man ihn wiederfinden konnte, um ihn dann zu verwenden. Das Buch, dessen Aufbau noch derselben (durch den Index locorum angezeigten) Regel gehorcht, bietet jedoch eine den inneren und zur Hälfte unbekannten Regionen zunehmend fremde Kartographie, in denen das begehrende Subjekt umherirrt. Der Leser muss daher mit den locides Buches spielen, muss sie mischen, Grenzen überschreiten, um die beweglichen und beiläufigen Figuren seiner Wege nachzuzeichnen, oder er muss annehmen, dass in ihm verborgen ein geheimes »Buch« existiert, ein unbekanntes, atopisches Gedächtnis, in dem die unbestimmten Figuren des Begehrens eine Unterkunft finden. Es gibt keine festgefügte Ordnung mehr zwischen den Figuren und den Orten.

11 4) Der Gebrauch des Buches erfolgt gemäß Bestrebungen, die nicht mehr auf eine Ordnung der Äußerungen abzielen, sondern auf die Möglichkeit einer Aussage. ${ }^{8}$ Im Akt des Sprechens zu oder des Hörens (mittels Gebet, Unterhaltung oder conversar) laufen die mystischen Vorgehensweisen zusammen. Das Buch erscheint nun als eine Art zu lernen, wie man (zu Gott) spricht oder (ihm) zuhört. Man geht davon aus, dass es die Vorstellung von einem Redeort hervorruft, der einmal der des Anderen, einmal der des Lesers ist. Es ist in einer dialogalen Problematik eingebunden: bald steht es für die Stimme des Senders, bald für die des Empfängers. Dieser Ort ist also, ob implizit oder explizit, zwischen zwei Formen des "Sagen-Wollens" aufgeteilt, die sich im geschlossenen Feld eines gelesenen und wie ein Stimmenraum praktizierten Textes gegenseitig suchen. In der mystischen Literatur hat er daher sehr häufig die Form des Dialogs angenommen.

12 5) Wenn schließlich das Buch für diese mystischen Leser noch teilweise der Sakramentalität der Dinge untersteht, insofern es von den "Wundern" und "Mysterien« des Schöpfers erzählt, kommt ihm diese Bedeutung doch nur noch aus seiner traditionellen Funktion zu. Es ist hier eher der kulturelle Horizont, von dem es sich abhebt. Die Dynamik seiner Herstellung und seines Gebrauchs verleiht ihm hinsichtlich seines Verhältnisses zu Gott entweder die Rolle eines Zeugnisses dafür, dass ein ursprüngliches Wollen existiert, das allen Dingen Bedeutung gibt (eine freilich nicht zu entziffernde Bedeutung, die sich nicht in "Mysterien« oder Glaubensartikel ummünzen lässt), oder die Rolle eines Anzeigers von Operationen, die vorgenommen werden müssen, um über die Identifikationen oder Lokalisierungen des Göttlichen hinauszugehen. Unter diesem doppelten Modus ist es Verweis auf ein Namenloses. Ihm obliegt immer weniger, die Geheimnisse Gottes lesbar zu machen, dagegen immer mehr, die für die mystische Erfahrung erforderlichen Praktiken des Leerwerdens zum Ausdruck zu bringen. Das Buch erzählt eher »Geschichten«, das heißt paradigmatische Aneinanderreihungen von ausgeführten oder auszuführenden Handlungen, als gnostische Systeme. Weil es weniger epiphanisch als technisch angelegt ist, dient das 
Buch viel mehr als ein Operator für theoretische Übungen denn zur Verkündung von Wahrheiten.

\section{Die »Momente» der Lektüre}

13 Wenn ich hier den historischen Ort der von den Mystikern bevorzugten Lektüre angebe, will ich damit schon ihre Besonderheit nahelegen. Das Buch fügt sich übrigens wie eine symbolische Hälfte an die Lesepraxis seiner Zeit an. Es verweist darauf. Es bezeichnet sie je schon. Dass das Buch auch über diese Lesepraxis hinaus Bestand hat, weiterhin aber deren Spuren aufweist, und dass das Buch später wieder durch Lektürepraktiken angegangen wird, für die es nicht konzipiert wurde, darin steckt auch eines der Probleme, die uns heute die Lektüre jener alten »Mystiker« bereitet. Man kann daraus zumindest einige Hinweise auf das Leseverhalten ziehen.

Die Informationen stellen sich als Geschichten über die Beziehungen des Lesers zum Buch dar. Eine zentrale Tatsache: dieses Verhältnis variiert mit den Etappen oder dem "Fortschreiten" des lesenden Subjekts, ist also nichts Stabiles. Es macht ganz unterschiedliche "Momente" durch. Diese sich auf den von einem Subjekt zurückgelegten Weg beziehenden strategischen Positionen lassen sich (etwas gewagt) in einem Schema wiedergeben, das die unzähligen Geschichten, die uns die Beziehungen der Mystiker zum Buch erzählen, zusammenfasst. Mit diesem Schema reduziere ich die Modalitäten dieser Beziehungen auf vier aufeinander folgende Positionen, die 1) den Beginn der Erfahrung betreffen, 2) das Buch als Garten der Lüste und Ort für deren Genuss, 3) die "Mandukation«, die auf einen "Gewinn« oder die Verfertigung eines symbolischen Körpers abzielt, und 4) eine Ablösung, durch die der Leser sich vom Buch entfernt und sich »erheben« kann, was dann der spezifische Moment der »absoluten« Lektüre ist.

\section{Das Beginnen oder die Einführung}

Lesen macht zuallererst ein Beginnen möglich. Es ist wie eine Schwelle: Sie ist nötig, damit es für die subjektive Suche ein Anderes gibt. Das Buch schafft so etwas wie ein Draußen. Es umreißt eine Alterität in dem weiten Feld, das im Ganzen genommen für eine »religiöse« Sprache gehalten wird, in dem ein Begehren herumirrt, das sich nicht kennt. In der Hauptsache liegt seine Bestimmung nicht in der Wissensvermittlung, sondern darin, in einer unübersichtlichen Landschaft, von der man annimmt, dass sich darin das Göttliche findet, die Unterscheidung hin zu einem undurchsichtigen Da-Sein $\mathrm{zu}$ ziehen. Es ist weniger die Aussage eines Signifikats, sondern ein Signifikant des Anderen. Seine Rolle besteht also zuallererst darin, einen Leser möglich zu machen, das heißt die Kontaktaufnahme einer Erwartung mit einer Objektivität, die ihr Widerstand entgegenbringt und von woanders kommt, die sich prinzipiell aber in der Region des Sinns verortet. Das Buch verknüpft eine innere Erwartung mit einem fremden Ort, der als Verweis auf ein anderes Sagen-Wollen angesehen wird. Innerhalb des unbestimmten Horizonts, in dem sich das Begehren bewegt, schafft es Teilung, die elementare Struktur und Bedingung der Möglichkeit für eine zukünftige dialogale Praxis. Es setzt die Unterscheidung, ohne die es keine Beziehung gibt. 
16 Warum ist diese Aufgabe gerade dem Buch übertragen? Mangels geeigneter Institutionen und Autoritäten nimmt es die Form einer gestaltgewordenen Alterität ein. Teresa von Ávila erklärt beispielsweise, dass sie in ihren Anfängen »einen Lehrmeister [...], der mich verstanden hätte«, nicht gefunden und daher keine andere Zuflucht gehabt habe als das Buch und dass ihre Seele, »wenn ich kein Buch hatte [...] gleich durcheinander [war] und meine Gedanken schweiften ab«. Zu dieser Rolle des Buches als Platzhalter des Anderen, indem es zur Fiktion (oder zum Ersatz [dt. im Orig.; A. d. Ü.]) einer Differenz wird, die eine Bewegung des Begehrens "gestattet «, fügt sie noch hinzu, dass man sich damit während der für das Reden oder die oración vorgesehenen Zeit beschäftigen kann (ocuparse mucho en lición): Es ist recreación. Da, wo Teresa aus Mangel an Phantasie, wie sie sagt, eine »Leere« oder geistige Zerstreutheit empfindet, erlaubt ihr das Buch ein Vokabular der Erwartung auszubuchstabieren und über die darin enthaltenen Worte $\mathrm{zu}$ wandeln, so als ob sie für die verworrene Vokalisierung ihrer Wünsche Konsonanten lieferten. Daher erklärt sie das Lesen als »sehr hilfreich«, »auch wenn [man] nur wenig lesen mag«. »In all diesen Jahren wagte ich nie, ohne ein Buch mit dem Beten zu beginnen, außer gleich nach der Kommunion.« ${ }^{9}$ Die adressierte Rede (das »Gebet«) vollzieht sich nicht ohne das Buch. Nicht ohne[deutsch im Orig.; A. d. Ü.]: Die Kategorie des »Nicht-ohne« bezeichnet die Voraussetzung für das Beginnen.

17 Teresa präzisiert noch, dass das Buch als Ding es ermöglicht, »herauszutreten« und zugleich zu »verbleiben«. Es lässt sie aus der Unbestimmtheit, in der sie sich befindet, heraustreten (während "aus ihr nichts herauskommt«) und in der Einsamkeit verweilen(während ihr Geist zum Umherschweifen neigt). Seine Bedeutung liegt daher darin, dass es einen notwendigen Baustein für eine mentale Physik der Erwartung darstellt. Was es einen tun lässt, zählt mehr als das, was es einem zu denken gibt. Bis zu einem gewissen Grad genügt seine Bedeutung als etwas Signifikantes. Hält man es in der Hand, beschwert es das Gebet und setzt es in Gang. "Oft«, sagt sie, »brauchte es nicht mehr, als das Buch zu öffnen ${ }^{10}{ }^{10}$. Dieses geöffnete, aber nicht gelesene Buch zeugt von einer Lesbarkeit des Anderen, ohne dass diese tatsächlich praktiziert wird. Wie alle Institutionen und an ihrer Stelle ist es das stumme Da-Sein des zweifachen Postulats, das ein Reden ermöglicht: Es gibt etwas Anderes, es gibt Sinn. Es reicht, dass das Buch wie ein Stein oder ein Altar einfach da ist, um dies zu gewährleisten. Das schiere Dasein ruft Worte hervor, die sich dann von selbst vervielfältigen.

Das Buch lässt sich im Übrigen auch durch einzelne Elemente des umgebenden Geländes ersetzen: ein fließendes Gewässer, Blumen usw., die in die Landschaft wie Schriftzeichen auf einer Seite eingeschrieben sind und damit zu »Platzhaltern des Buches « werden. ${ }^{11}$ Ein Jahrhundert früher rieten Seuse, Tauler und andere dem Betenden, das Gebet auf "natürliche« Dinge zu stützen oder zu heften, aber sie hatten für sich selbst Bedeutung als von Gott gegebene Zeichen. Mit Teresa wird das Buch zum Referenten. Da die Blüten den Buchstaben eines aufgeschlagenen, aber nicht gelesenen Buches ähneln und weil sie derart das schweigende Sakrament einer Lesbarkeit darstellen, können auch sie etwas zur Sprache bringen. Doch letztlich ist es das Buch, das - in der Art einer stillschweigenden Voraussetzung oder einer stumme Institution zum Reden veranlasst. 


\section{Der Garten der Träume}

19 Eine sehr weit zurückreichende Tradition zeugt davon, dass das Buch dem Leser einen Raum des Genusses zugänglich macht. „Sapor et non scientia", schrieb Bonaventura im 13. Jahrhundert. ${ }^{12} \mathrm{Zu}$ Beginn des 17. Jahrhunderts wiederholt Alvarez de Paz: »Non notitiam, sed gustum et affectum « ${ }^{13}$, und Ende des vorangehenden Jahrhunderts fasst Luis de Granada, ein großer Sammler mystischer Literatur, sie zusammen, indem er vom »Reiz« und "Genuss" des Lesens spricht. ${ }^{14}$ Der beim Gebrauch des Buches bevorzugte gustus, der sapor und die inflammatio usw. bedeuten ein "Genießen« und ein "Schmecken«. Doch diese affektive Besetzung erfordert eine Mobilisierung des Willens. Emotion und Antrieb (motion) hängen eng zusammen. Der affectus verweist auf den motus, auf eine actio des Äußerns und der Performanz.

Ausgangspunkt dieser aktivierenden Ergriffenheit ist ein früher schon vorhandenes Vergnügen. Einmal mehr ist es Teresa, die sagt: »Immer habe ich den Wunsch, Zeit zum Lesen zu haben, da ich immer sehr daran gehangen habe. ${ }^{15}$ Seit den Ritterromanen und den Heiligenviten ihrer Jugend liest sie mit »Genuss « und »Hingabe « ${ }^{16}$ In ihren einsamen Reisen geht es nicht um Wissenserwerb. Diese folgen Erzählungen von (Ritter-, Helden-, Liebes- und spirituellen) Aventiuren, deren einzelne Etappen eine Topographie der Innerlichkeit zeichnen. Es sind Wanderungen in eine Erinnerung, in der sie unbekannte Orte enthüllen, so wie ein Gedicht nebenbei schlummernde Feen weckt. Diese Lektüre hat also Ähnlichkeit mit dem Traum, der mit Tagesresten Dinge erzählt, die sich am Tage verbieten. Bei Teresa kommt der »Traum« von überall her und verweist auf dieses andere Leben, das weniger diskret "spirituell« heißt. Die biblische Allianz von Traum und Geist. »Und er [der Herr; A.d.Ü.] hat mein Leben zu einer Art Traum gemacht, so daß ich fast immer den Eindruck habe, daß ich das, was ich erlebe, nur träume. ${ }^{17}$ Lición und sueño gehen hier Hand in Hand. Das Lesen ist eine Lehre im Träumen. Es verleiht ihm auch Legitimität. Es gestattet die Kühnheit, daran zu glauben. Es sagt: »Nehmt eure Wünsche für die Wirklichkeit.« Es schafft für sie einen Spielraum, eine Landkarte vergangener oder möglicher Wege. Denn das Buch hat daraus schon Ritter- und Heiligengeschichten gemacht, spirituelle Aufstiege und mystische Suchfahrten. Es gestaltet so Gärten, in denen das unbewusste »Innere« Wege für seine Heiterkeit, seine Glut und seine Traurigkeiten findet.

21 In der Tradition des klösterlichen Paradieses (paradisus claustralis) und der Gärten der feinen Liebe ${ }^{18}$ - einer von Teresa zu Beginn der Moradas mit der Beschreibung des BurgKristall-Paradieses, das sich ihr als theoretisches Theater »dargeboten« hat, in dem sich die gesamte mystische Reise der »Seele« darstellt, wiederaufgenommenen Tradition ${ }^{19}$ ist das Buch der neue umfriedete Garten, der Wandelgang einer »Einsamkeit«. Die Lektüre treibt dabei zwischen dem niedergeschriebenen Traum und dem geträumten Geschriebenen ein Doppelspiel: Sie geht hin und her zwischen dem Traum, der die objektive Form einer niedergeschriebenen Geschichte hat, und dem Traum, der sich wie ein durch das Vorübergehen dieser Geschichte aufgeweckter Geist an die Erfindung seiner Reise macht. Der Konvent, wie ihn sich Teresa entwirft, wird eine Wiedergabe dieses Buches sein, ein geschlossener Ort, dessen fiktive Existenz es den "Liebhaberinnen Gottes« möglich macht, frei zu promenieren.

22 Folglich ist die Fiktion nicht nur für den Text kennzeichnend, insofern er eine Hervorbringung des Begehrens ist. Seine Lektüre bewirkt ihrerseits die Fiktion eines Textes. Sie geht selbständig über den Schreibvorgang hinaus. Ihr Theater verdoppelt 
jenes, das ihr selbst als Initiierung gedient hat. Sie richtet ihre eigenen Orte ein. "Gefallen am Text.« Diese Lektüre entbehrt allerdings der von ihr geformten Objekte. Was sie hervorbringt, entzieht sich ihr und bleibt eine von flüchtigen Boten bevölkerte imaginäre Welt. Das Spiel des Genießens ist zugleich das eines Verlusts. Die Erfahrung strebt auf das Nichts dessen zu, was sie sich ver-gegenwärtigt. Sie übt sich in dem, was ihr fehlt. Da der Affekt auf den Ort verweist, von wo aus man spricht, tritt er freilich aus einem Äußerungsplatz heraus: Genuss des Sagens. Doch wie in der melancholischen Struktur (die philosophische und mystische Melancholia ${ }^{20}$ ), sagt (sich) das Subjekt nur, indem es sich von den Aussagen und Dingen ablöst. Das narzisstische Vergnügen, (von sich) zu sagen, macht aus der Äußerung einen leeren Ort. Der Welt enthoben durch die Trunkenheit des "Sagen-Wollens«, entbehrt der Leser eines Körpers. Er ist ekstatisch, durch ein Übermaß an In-Sich außer sich.

\section{Fiktionen von sprechenden Körpern}

Auch hier ist die spirituelle Tradition fordernd: »Nimm und iss«. Der Leser soll dahin kommen, den Text »wiederzukäuen", »weich zu machen«, zu »verdauen«, und damit von der sagenden Mündlichkeit zur essenden Mündlichkeit überzugehen. Die ruminatio der Kuh dient als Vorbild. So sagt etwa Wilhelm von Saint-Thierry - um nicht noch weiter zurückzugehen - im 12. Jahrhundert: "De quotidiana lectione aliquid quotidie in ventrum memoriae demittendum est, quod fidelius digeratur, et sursum revocatum crebrius ruminetur. ${ }^{21}$ Bis ins 17. Jahrhundert ist die Lektüre auch eine Sache des Mundes. Surin fordert, dass die Bücher-»Nahrung" solide und den "Notwendigkeiten" des »Magens der Seele« angepasst « sein soll. ${ }^{22}$ Das Essen des Buches kann zudem Krankheiten hervorrufen, bewirken, dass der Körper zunimmt oder in eine gute Form kommt. Es übt einen Reiz auf die Höhlen und Schleimhäute des Geistes aus und tut ihnen gut. Das Buch als Nahrungsmittel ist ein Fremdkörper, der ein inneres Sinnesvermögen visitiert, »in Ordnung bringt« oder auch verletzt. Intimes Ringen.

Nach dem zeitgenössischen Körperbild modelliert, verweist diese Physiologie der verdauenden Lektüre häufig auf die Topographien der physischen Orte des Geistes und auf die Alchemie der "sympathetischen Aktionen« zwischen ihnen. Doch welche Theorien auch immer man zugrunde legt ${ }^{23}$, diese Lektüre bringt einerseits die Beziehung zwischen Text und Körper zum Ausdruck, andererseits verknüpft sie die Begriffe davon mittels eines »Gewinns«. Zwei gewichtige Fragen stellen sich.

Die Erste stellt die Organisation des Körpers durch Sprachliches oder, besser gesagt, durch eine Schichtung von gebrochenen Sprachen in Frage. Wie die Informationen, von den Hysterisierungsphänomenen bis hin $z u$ den Determinierungen der Körper entsprechend der jeweiligen kulturellen Systeme, deutlich zeigen, ist der Körper das Resultat eines Zusammenspielstextueller Elemente wie zum Beispiel eine ausgeschmückte Geschichte, die durch die Spuren, welche Zitate des Signifikanten darin hinterlassen, geschrieben und aufpoliert wird. Die »verdauende« Lektüre zeugt dann entsprechend noch davon, wie Teile des Buches noch an diese körperlichen Markierungen gebunden sind. Es sieht ganz so aus, als ob diese Lektüre eine innere Haut für die Tätowierung aufwiese, die dieses oder jenes Wort entsprechend der "Sympathien «, die der Repräsentation des Körpers oder der des Textes entgehen, darin unvermittelt einritzt. Derart kommt es zu textuellen Implantationen irgendwo im 
»Magen«, zu Schmerzen, Darmverschlingungen oder -erweiterungen, aber auch zu Herzwärme, zu Armbewegungen, zu Tränen... »Ich weine, weil es so gut gesagt ist.«

Man richtet sich darauf durch eine langsame, fast schon tastende Lektüre aus, die dort, wo man »kostet", innehält, das heißt, wo sich erfahrungsgemäß eine dieser "sympathetischen Aktionen« zeigt. Man muss also notwendiger Weise die Menge an Gelesenem verringern, um diese Markierungen zu intensivieren. »Wenige Dinge, die in die Tiefe gehen, bringen mehr Gewinn als viele, die nur gestreift werden«, schreibt Surin. ${ }^{24}$ Im äußersten Fall genügt ein Wort, God, Love ${ }^{25}$, ein verbaler Pfeil in einem bebenden Leib.

Der "Gewinn" ist die Wirkung dieser Berührung, ein Gleichklang zwischen einem bestimmten Textduktus und einer Körperpartie. Schrift des Körpers oder Inkarnation des Textes - er hat von beidem etwas. Er stellt eine partielle Fiktion (oder Fabrikation) des "symbolischen « Körpersher. Der opake Ort der physischen Erfahrung hallt bei einem unvorhergesehenen Detail des Geschriebenen in einem Punkt wider. Es ist eine an einen Ort gebundene Genese: "Aufstöhnen«, Tränen, Aufwallen oder Beklemmungen der Brust, Rumoren oder Bewegung im Innern. Etwas kommt zur Sprache, das weder das Subjekt noch das Buch, sondern deren Entstellung ist, das Zusammentreffen selbst, eine "Liebesmüh«. "Ich weine ohne Grund, den ich euch nennen könnte, es ist wie ein Kummer, der durch mich hindurchgeht, es muss ja doch wohl jemand weinen, es ist, als wäre ich es.«"26

Als edificatoria lectio ${ }^{27}$ »erbaut « diese Lektüre nämlich ein Sprechkörper-Fragment. So wie sich ein Ort in einer Landschaft beleben würde und dabei den dort befindlichen Geist zum Ausdruck brächte, entspringt eine Stimme aus dem Körper, ohne ihm anzugehören, ohne dass man wüsste, warum gerade da, und ohne dass man das benennen könnte, was spricht. Da in eine "Fabel« verwandelt, wo er vom Text angerührt wird, wird der Körper zum Echo einer hinter dem Geschriebenen abwesenden Rede. Er tritt an ihre Stelle wie ein Stück sprechender Text, wie ein Stimmenzitat.

\section{Die Unterbrechung und der gebrochene Text}

Wilhelm von Saint-Thierry empfahl die "oratio quae lectionem interrumpat «. ${ }^{28}$ Dieser Moment der Loslösung wird durch die Lektüre selbst vorbereitet. »Richtet die Augen fest auf das Buch, heftet jedoch nicht euer Herz daran ", wie Johannes von Ávila sagt. ${ }^{29}$ Eine diffuse Aufmerksamkeit wahrt einen Horizont an Absolutem. Sie darf nicht in dem abgeschlossenen Bereich des Textes zurückgehalten werden. Sie entschlüpft bereits in ein Hinterland und die Texte sind dabei eigentlich nur Durchgangsorte - hier ist es nicht, da ist es nicht -, die man gleich wieder verlassen muss. Es kommt also zu einem Bruch. "Man tut gut daran, die Lektüre zu unterbrechen «. ${ }^{30}$ Ein allgemeingültiger Ratschlag. Man muss diese Verbindungen kappen, damit der Leser eine "nie gesagte" Rede vernimmt, so wie Don Juan mit den Frauen bricht, um die Frau zu suchen. Ein Kommentator geht soweit $\mathrm{zu}$ schreiben: »Das beste Buch ist jenes, das am vollständigsten verschwindet. « ${ }^{31}$

Der Text unterliegt dem Gesetz des Nicht-zuviel. Er wird schnell übermäßig, unanständig. Das Begehren ist klaustrophobisch, es kommt um durch ein Übermaß an Bücherpräsenz und durch die Einsperrung in den Sinn. Eine echte Lektüre wird Tony Duvert zufolge »beim ersten Sagen der Fremdheit entdeckt«. Diese »Lesbarkeit macht 
das Werk dunkel, weil sie um der niemals gesprochenen Sprache eines nie gesagten Wirklichen - des Köpers, des Objekts, des Nicht-Sinns - auf das gesellschaftlich vorgeschriebene Kommunizierbare verzichtet «. ${ }^{32}$ Dieses sich auf das Andere richtende abweichende "Lesen« scheint im 16. Jahrhundert nur durch das Verzichten auf den Text möglich zu sein, als ob etwas, das der Text vorbereitet hat, direkt den Körper markieren musste, als ob nach der durch die Lektüre eines Buches ermöglichten entstellenden Übung eine unbekannte Hand (ein Engel oder gar Gott selbst?) selbst die Schriftzeichen der Liebesbesessenheit auf sämtliche Mauern dieses zum Warten erweckten Hauses zeichnen konnte.

31 Wie es sich auch immer mit diesem Nicht-Text verhält, zu dem der Leser des Textes übergeht, dieser Übergang bewirkt ein zunehmendes In-Stücke-Reißen des Buches bis hin zu seinem schließlichen Vergessen. Das Buch wird zerstückelt, zerschnitten und zerstampft. Es bleiben »Blütenlesen«, Exzerpte - Auszüge, Zitate und Überreste von ein paar Wörtern Länge, deren Wiederholung den Sinn ausschöpft und die, vermischt mit anderen, ein Kontinuum ohne Sprecher oder Autoren, eine sprachliche Magma ergeben, wodurch sich mit Sprachlichem eine Redestelle andeutet. Diese noch aus einer aufgegebenen Geschichte zutage tretenden verstreuten, geschichteten Textruinen scheinen auf eine Verödung der Landschaft bezogen zu sein, aus der sich der Mystiker entfernt. Verarmung, ja Taumel gehen aufgrund von Unsicherheit (das heißt angesichts von einer Brüchigkeit zur nächsten) mit diesem Fremdwerden einher. Bald überlebt wie ein letztes Metrobillet in der Tasche des Emigranten im Geiste nur noch eine Gedichtzeile. Und wenn durch eine stets mögliche Umkehr der Betende diese Reliquie nicht fetischisiert, dann vergisst er sie schließlich. Er ist auf hoher See.

Es kommt offenbar zu einer widersetzlichen Erfahrung. Sie bringt diese Fragmente dazu, über sich hinauszugehen, anstatt sie aufzulösen oder auszuwischen. Dadurch, dass sie sie an sämtliche Umstände "anpasst«, macht sie sie allgegenwärtig. Wenn Francisco de Osuna von DER Schrift spricht, die »mehrere Gesichter« hat und die »ein jeder nach Belieben passend machen kann " - ein wie Stoff beweglicher und flexibler Text -, vergleicht er sie mit »Personen auf manchen Malereien«, die »den Betrachter immer wieder dorthin nachzublicken scheinen, wo er sich hin begibt « ${ }^{33}$ Wie ein solcher Blick folgt der Textsplitter dem Passanten überall hin. Wie auch das Fragment einer Skulptur, der »Archaïsche Torso Apollos« bei Rilke, ist der seines Hauptes und seiner Bedeutung beraubte Textbrocken ein »Kandelaber, in dem sein Schauen, nur zurückgeschraubt, sich hält und glänzt«:

»denn da ist keine Stelle,

die dich nicht sieht. Du mußt dein Leben ändern. « $^{34}$

Woher kommt diese Forderung? Wie das Orakel spricht das Textfragment weder die Wahrheit noch die Unwahrheit, es »bedeutet«, wenn es den Wanderer anstarrt, mit einem nun nicht mehr nur in Büchern verfangenen Blick. Dieser Blick ist der des Anderen. Was gerade noch Objekt, was gerade noch widerständige Alterität war, hat sich in ein unsichtbares Subjekt verwandelt. Das Objekt verschwindet, vergessen, wie eine Sache, die in ein Zeichen für das, was der Deutung oder der Handhabung entgeht, verwandelt wurde, und zielt nun auf »dein Leben«. 


\section{Wo soll man das «Werk« lesen?}

In der mittelalterlichen Folge oder religio, die von der lectio zur meditatio führte, und von dieser zur oratio, dem Weg zur contemplatio ${ }^{35}$, die also vom Anhören des Meisters (was der Lektüre entspricht) zur Reflexion verlief, schließlich zur An-Rede, bevor sie zur "Weisheit« gelangte ${ }^{36}$, scheint es zu einem Riss der Kette, zu einem Durchtrennen ihrer Glieder gekommen zu sein. Das Buch spricht nicht mehr, ebenso wenig vernimmt es sich. Die "Besinnung" hat etwas mit Verstehen zu tun, das die Mystiker beinahe einhellig im Verdacht haben, dass es ein Eigentümer-Wissen darstelle. Die Rede hallt in einem stimmenlosen Universum wider und an ihre Stelle treten diese Körpergeräusche, über deren Sprecher es keine Gewissheit gibt. Die aus ihrer Umlaufbahn getretene "contemplatio" sucht im "Sinnlosen" oder "Außergewöhnlichen" die mit Verrücktheiten gesäumten Wege, was nur eine Änderung - ein Herzleid - garantiert.

Das Buch, das den Platz der Institution und des Meisters einnimmt, wird ebenfalls aufgegeben. In Augustine Bakers Sancta Sophia (1657) sind die neuen »Herren« nur noch "Diener « ${ }^{37}$ Die Kontemplation lässt sie in Absetzung zur gesamten monastischen Tradition des Mittelalters hinter sich. ${ }^{38}$ Schließlich liefert die Lektüre der »himmlischen Philosophie« nur »ein Alphabet ${ }^{39}$, kleine auf einem ungeebneten Weg verstreute Steinchen. Es ist nicht so, dass das Lesersubjekt sich davon löst, dass es autonom wäre. Es weiß nicht länger, wo es ist noch was es ist.

Vielleicht gibt es in dieser Unentschiedenheit zwischen einem von seinem Unwissen gespaltenen Subjekt und dem zerrissenen Buch, das nach und nach um eines Anderen willen vergessen in der Ungewissheit einer eindringlichen Forderung (»Du musst dein Leben ändern«) verändert wurde; vielleicht gibt es in dieser Unentschiedenheit eine Lektüre, die zu jener führt, von der Blanchot spricht: »Das Buch ist also da«, sagte er, "aber das Werk ist noch verborgen, vielleicht radikal abwesend, in jedem Fall verschleiert, kaschiert durch die Offenkundigkeit des Buches, hinter der es auf die befreiende Entscheidung, das Lazarus, veni foras ${ }^{40}$ wartet." Man müsste sich demnach fragen, welche Hoffnung auf »etwas anderes«, wie sie im 16. Jahrhundert aufkam, dazu führte, das durch das Geschriebene angekündigte/verschleierte »Werk« außerhalb des Buches, beim sprechenden Körper anzusetzen; ebenso, welche zeitgenössische Modifikation seit Mallarmé diese Hoffnung untersagt und »dieses Grabmal « ${ }^{41}$ auf ein »Diesseits« oder ein »Jenseits« des Verstehens des Geschriebenen zurückführt und die Hypothese einer von anderswo her kommenden oder gar im Buch herumgeisternden "Stimme« (für wen und für wie lange?) bestätigt. Diese Hypothese wäre heute der Mythos der Lektüre, dasjenige, was sie weder erreichen noch preisgeben kann.

\section{BIBLIOGRAPHIE}

Baker, A. (1953): La Sainte Sapience, Paris (dt. Übers.: Heilige Weisheit. Eine Anleitung zum mystischen Gebet aus dem 17. Jahrhundert, St. Ottilien (Orig.: Sancta Sophia, Douai 1657). 
Blanchot, M. (1955): L’Espace littéraire, Paris.

Brillet, G. (1945): »La Bible et la lecture spirituelle«, La vie spirituelle, Bd. 73.

Comito, T. (1978): The Idea of the Garden in the Renaissance, New Brunswick (N.J.).

de Certeau, M. (1976): »L'énonciation mystique«, Recherches de science religieuse, 64, S. 183-215

de Certeau, M. (1978): »Mélancolique et/ou mystique«, Analytiques, 2, S. 35-48.

de Certeau, M. (1980): L'invention du quotidien, Bd. 1: Arts de faire, Paris.

de Granade, L. (1863): Euvres complètes, Paris.

de Lubac, H. (1964): Exégèse médiévale. Les quatre sens de l'Ecriture, Bd. 4 = 2,2, Paris (teilweise übers. in: de Lubac, H.: Typologie, Allegorie, geistiger Sinn. Studien zur Geschichte der christlichen Schriftauslegung, übers. und eingel. von R. Voderholzer, Freiburg i. Br. u. a., 1999).

de Ros, F. (1936): Un maître de sainte Thérèse. Le père François d'Osuna. Sa vie, son oeuvre, sa doctrine spirituelle, Paris.

Debus, A. G. (1977): The Chemical Philosophy, 2 Bde., New York: Science History Publications.

Duras, M. (1984): Der Vize-Konsul, Frankfurt (frz. Orig.: Paris, 1966).

Duvert, T. (1972): »La lecture introuvable«, Minuit, 1, S. 2-21.

Eisenstein, E. L. (1969): »The advent of printing and the problem of the Renaissance«, Past and Present, 45, S. 19-89.

Furet, F./Ozouf, J. (1977): Lire et écrire. L'alphabétisation des Français de Calvin à Jules Ferry, 2 Bde., Paris: Minuit.

Gerson, J. (1960): Cuuvres complètes, Bd. 2: L'œuvre épistolaire, hg. v. P. Glorieux, Paris.

Guillaume de Saint-Thierry (1862): »Epistola seu Tractatus ad Fratres de Monte Dei«, I.10, 31: Patrologia Latina, 184, 327d (dt. Übers.: Wilhelm von Saint-Thierry (1992): Goldener Brief. Brief an die Brüder vom Berge Gottes, übers. v. B. Kohout-Berghammer, Eschenbach).

Hébrard, J. (1979): „École et alphabétisation au XIXe siècle«, http://www.persee.fr/web/revues/ home/prescript/article/ahess_0395-2649_1980_num_35_1_282610 [Stand: 18.3.2012]

Jean d'Avila (= Juan de Ávila = Johannes von Gott) (1954): Audi filia, Paris.

Johannes vom Kreuz [= Juan de la Cruz] (2007): Aufstieg auf den Berg Karmel. Vollständige Neuübersetzung, hg. v. U. Dobhan, E. Hense u. E. Peeters, in: ders: Gesammelte Werke, Bd. 4, Freiburg.

Leclercq, J. (1957): L'amour des lettres et le désir de Dieu. Initiation aux auteurs monastiques du Moyen Âge, Paris.

Lepée, M. (1951): Sainte Thérèse mystique: Une divine amitié, Paris.

Massero, D. W. (1975): Understanding Language, New York: Academic Press.

Melher, J. /Noizet, G. (Hg.) (1973): Textes pour une psycholinguistique, Paris, den Haag: Mouton.

o. V. (1982): The Cloud of Unknowing and Related Treatises (Analecta Cartusiana 3), Salzburg (dt. Übers.: Die Wolke des Nichtwissens (Christliche Meister, Bd. 8), hg. v. W. Riehle, Einsiedeln, 1999).

Rilke, R. M. (1918): Der neuen Gedichte anderer Teil, Leipzig.

Sieben, Hermann Josef, SJ (1976): Art. »De la lectio divina à la lecture spirituelle«, in: Dictionnaire de Spiritualité, Bd. 9, S. 487-496.

Surin, J.-J. (1659): Catéchisme spirituel, Paris. 
Surin, J.-J. (1963): Guide spirituel, hg. v. M. de Certeau, Paris.

Teresa von Ávila (2004): Gedanken zum Hohenlied, Gedichte und kleinere Schriften [Relación]. Vollständige Neuübertragung, hg. u. übers. v. U. Dobhan u. E. Peeters, in: dies.: Gesammelte Werke, Bd. 3, Freiburg.

Teresa von Ávila (2007): Wohnungen der Inneren Burg [Moradas]. Vollständige Neuübertragung, hg. u. übers. v. U. Dobhan u. E. Peeters, in: dies.: Gesammelte Werke, Bd. 4, Freiburg.

Teresa von Ávila (2009): Das Buch meines Lebens. [Libro de la Vida]. Vollständige Neuübertragung, hg. u. übers. v. U. Dobhan u. E. Peeters, in: dies.: Gesammelte Werke, Bd. 1, Freiburg.

Yates, F. (1990): Gedächtnis und Erinnern. Mnemonik von Aristoteles bis Shakespeare, Weinheim.

\section{NOTES}

1. Vgl. de Certeau (1980), Kap. 12.

2. Furet/Ozouf (1977). Der zweite Band enthält von F. Furet und J. Ozouf geleitete Regionalstudien zu Frankreich.

3. Vgl. die Einführung von Melher / Noizet (1973) oder Massero (1975), »Reading«, S. 207-311. Vgl. auch Hébrard (1979).

4. Johannes vom Kreuz (2007), Vorrede.

5. Teresa von Ávila (2009), Kap. 4.

6. Vgl. Eisenstein (1969) zum Buchdruck als einem »Epoche machenden Ereignis«.

7. Yates (1990).

8. Vgl. de Certeau (1976).

9. Teresa von Ávila (2009), S. 110. Charakteristische Einschränkung: wie das Buch wird das Brot der Kommunion mehr zum Objekt des Sinns als dass es selbst Sinn macht. Es artikuliert ein unbestimmtes Verlangen durch eine Unterscheidung von Subjekt und Objekt. Die Parallelität von Brot und Buch ist übrigens nichts der Tradition Fremdes.

10. Teresa von Ávila (2009), S. 111.

11. Teresa von Ávila (2009), Kap. 7.

12. [»Geschmack und nicht Wissenschaft«; A.d.Ü.]

13. [»Nicht Wissen, sondern Schmecken und Empfindung«; A.d.Ü.]

14. Vgl. beispielsweise die bei Sieben (1976) zitierten Texte.

15. Teresa von Ávila (2004), S. 199. Vgl. Lepée (1951), S. 314.

16. Teresa von Ávila (2009), Kap. 1, 2, 4, 6, 8 usw.

17. Teresa von Ávila (2009), Kap. 40.

18. Vgl. Comito (1978), Kap. 2-4.

19. Teresa von Ávila (2007), Kap. 1,1. Dieser Text nimmt im Übrigen in eher synthetisierender Gestalt den Vergleich mit dem Garten wieder auf, der in Teresa von Ávila (2009), Kap. 11-19 als Rahmen für die Analyse der Kontemplation dient.

20. Vgl. de Certeau (1978).

21. Guillaume de Saint-Thierry (1862), I.10,31: PL 184, 327d [»Von der täglichen Lektüre ist jeden Tag etwas in den Magen der Erinnerung abzuführen, auf dass es getreulich verdaut werde und sich, wieder nach oben befördert, häufiger wiederkäuen lässt«; A.d.Ü.].

22. Surin (1659), IV, 2 (ms).

23. Debus (1977).

24. Surin (1963), IV, 3.

25. The Cloud of Unknowing (1982), Kap. 7.

26. Duras (1984), S. 191. 
27. Ausdruck aus dem gegen Ende des 15. Jahrhunderts von einem Schüler Richard Rolles verfassten Speculum inclusorum.

28. Guillaume de Saint-Thierry (1862), I.10,31: P.L. 184, 328a [das Gebet, das die Lektüre unterbreche; A.d.Ü.].

29. Jean d'Avila (1954), S. 195.

30. Jean d'Avila (1954), S. 195.

31. Brillet (1945), S. 498.

32. Duvert (1972), S. 11.

33. Zitiert nach de Ros (1936), S. 396.

34. Rilke (1918), S. 1.

35. [lectio (Lesung), meditatio (Besinnung), oratio (Gebet), contemplatio (Gottesschau); A.d.Ü.]

36. Gerson, Brief an Guillaume Minandi, 30. Oktober 1422, in: Gerson (1960), S. 235; vgl. de Lubac (1964), S. 492.

37. Baker (1953), Bd. 1, S. 68.

38. Vgl. Leclercq (1957).

39. de Granada (1863), S. 143-144.

40. Blanchot (1955), S. 203 [Lazarus, komm heraus! Johannes 11,43; A.d.Ü.].

41. Das sind Blanchots Worte, (1955), S. 202-206.

\section{INDEX}

Mots-clés : lecture absolue, livre, mystique, écriture, rêve

Schlüsselwörter : absolute Lektüre, Buch, Mystik, Schreiben, Traum

\section{AUTEURS}

\section{MICHEL DE CERTEAU}

Michel de Certeau (1925-1986), Philosoph und Historiker. Nähere Informationen finden Sie hier. 